\title{
FINITENESS IN PRIME IDEALS IN RINGS OF GLOBAL DIMENSION TWO
}

\author{
HU SHENG ${ }^{1}$
}

\begin{abstract}
Let $A$ be a commutative ring with identity. The main result states conditions that ensure the finiteness of prime ideals in a coherent ring $A$ of global dimension two. Precisely, any ideal containing two noncomparable prime ideals is finitely generated. As a corollary it follows that a Krull domain of global dimension two is noetherian. Another corollary is that if $A$ is not semihereditary it contains a finitely generated maximal ideal.
\end{abstract}

Introduction. The purpose of this paper is to consider properties of finiteness of ideals in rings of global dimension two, in particular, the finiteness of prime ideals in a coherent ring of global dimension two. The main result states that any ideal in $A$ containing two noncomparable prime ideals is finitely generated if $A$ is a coherent ring of global dimension two.

As a corollary it follows that a Krull domain of global dimension two is noetherian. Another amusing consequence is that if $A$ is not semihereditary it contains a finitely generated maximal ideal.

Next we show that if $P$ is a finitely generated nonminimal prime ideal of a ring $A$ of global dimension two, $A / P$ is noetherian and $P$ can be generated by three elements, which is the same estimate obtained in the classical noetherian case.

1. Preliminaries and statement of main result. Any unexplained terminology is either standard (as in, for example, [6]) or defined in [11].

Let $A$ be a commutative ring with identity. A ring is said to be coherent if all of its finitely generated ideals are finitely presented. An ideal $I$ is finitely presented if there is an exact sequence

$$
0 \rightarrow K \rightarrow F \rightarrow I \rightarrow 0
$$

where $F$ is free and both $F$ and $K$ are finitely generated.

Presented to the Society, January 26, 1973; received by the editors April 2, 1973. AMS (MOS) subject classifications (1970). Primary 13B25; Secondary $13 \mathrm{C10}$.

Key words and phrases. Coherent ring, Krull domain, global dimension, prime ideal.

1 The results here are contained in the author's doctoral dissertation. The author is deeply indebted to $\mathrm{W}$. Vasconcelos for his help and encouragement in the preparation of this paper.

(c) American Mathematical Society 1973 
For an $A$-module $E$, the projective dimension of $E\left(\mathrm{pd}_{A} E\right.$, for short) is finite and equal to $n$, we recall, if there is an exact sequence

$$
0 \rightarrow P_{n} \rightarrow \cdots \rightarrow P_{0} \rightarrow E \rightarrow 0
$$

with $P_{i} A$-projective and $n$ least; otherwise the projective dimension of $E$ is said to be infinite.

The global dimension of $A$ (gldim $A$, for short) is then the supremum of the projective dimensions of all $A$-modules.

In [14] the main result here was proved for domains. As it happens, domains of global dimension two are coherent rings. It is thus natural to ask whether the result is still true when $A$ is coherent of global dimension two. By modifying the argument of [14], we prove the following theorem.

THEOREM 1.1. Let $A$ be a coherent ring of global dimension two and let $M$ be an ideal of $A$ containing two noncomparable prime ideals $P$ and $Q$ (i.e. $P \neq Q$ and $Q \not P$ ). Then $M$ is finitely generated.

We start with some basic lemmas.

LEMma 1.2. If $A$ is coherent of global dimension two, then the annihilator of each element a of $A$ is generated by one idempotent $e$. Furthermore, $a+e$ is a regular element of $A$.

Proof. Let $I=(0: a)=\{r \in A: r a=0\}$ be the annihilator of $a$. According to [10], if we can show that $I$ is finitely generated and $I^{2}=I$, then $I$ is generated by one idempotent. Since there is an exact sequence

$$
0 \rightarrow I \rightarrow A \rightarrow a A \rightarrow 0
$$

and $A$ is coherent, $I$ is finitely generated. By [7], $2=\operatorname{gldim} A \geqq \operatorname{gldim} A_{P}$, for prime ideal $P$ of $A$; on the other hand it follows from [11] that $A_{P}$ is a domain. Hence for each prime ideal $P, I_{P}=(0)$ if $I \subset P$ or $I_{P}=(1)$ if $I \not \subset$, thus giving $I^{2}=I$.

Let $I=(0: a)=e A$; if $r(a+e)=0$, we must show $r=0$, i.e. $a+e$ is a regular element. Since $0=0 e=(r a+r e) e=r e^{2}=r e, r a=0$ and $r \in A e$; therefore we can write $r=s e$ for some $s \in A$. Thus $r=s e=s e^{2}=r e=0$.

LEMMA 1.3. Let $I$ be an ideal containing a regular element of $A$, then $I^{-1}=\{x \in K=$ ring of quotients of $A: x I \subset A\}=A$ if and only if $I$ is overdense (i.e. $\operatorname{Hom}_{A}(I, A) \cong A$, under. the natural map).

The following lemma is Corollary 2.3 in [12].

LEMMA 1.4. Let $A$ be coherent with global dimension not bigger than two. If $I$ is an overdense finitely generated ideal of $A$; then $A / I$ is an artinian ring. In particular, the ideals above I are finitely generated. 
The following proposition is known as Burch's theorem [6, p. 148, Exercise 8] which we quote for convenience.

Proposition 1.5. Let $A$ be a commutative ring, $I$ be a finitely generated ideal with a resolution $0 \rightarrow A^{n-1} \stackrel{\theta}{\rightarrow} A^{n} \rightarrow I \rightarrow 0$. If I contains a regular element, then $I=t D$ where $D=\left(d_{1}, \cdots, d_{n}\right)$ is the ideal generated by all $n-1 \times n-1$ minors of the $n \times n-1$ matrix corresponding to $\theta, t$ is a regular element of $A$. Furthermore, $D^{-1}=A$.

\section{The proof of Theorem 1.1 .}

Proof. Since $P, Q$ are two noncomparable prime ideals contained in $M$, let us pick elements $a \in P-Q$ and $b \in Q-P$. According to (1.2), let $(0: a)=e A,(0: b)=g A$ where $e, g$ are idempotents of $A$. Let $I$ be the ideal generated by $a, b, e$ and $g$. Resolve this ideal in the following way

$$
0 \longrightarrow L \longrightarrow A^{4} \stackrel{h}{\longrightarrow} I \longrightarrow 0
$$

where $h(x, y, z, w)=a x+b y+e z+g w$. Since gldim $A=2$, [1] says that $\operatorname{pd}_{A} I \leqq 1$; and thus $L$ is projective. $L$ is also finitely generated for $A$ is coherent. Since $L$ is a finitely generated projective module, according to $\left[2\right.$, p. 138] there is a partition of the unity $\left(f_{1}, \cdots, f_{n}\right)=A$ with the property that $L_{f}$ is $A_{f}$-free of finite rank. (Here $f$ stands for any of the $f_{i}$ 's and $L_{f}$ is the module of fractions arising out of the multiplicative set of powers of $f$.) Again by [2, p. 137], we can prove $M$ is finitely generated by proving $M_{f}$ is finitely generated in $A_{f}$ for those $f \notin M$. Notice that in these cases the choices of $a, b, e$ and $g$ are not affected. Thus change notation and assume that $L$ is a free $A$-module of rank 3. Thus (*) becomes the following exact sequence

$$
0 \rightarrow A^{3} \rightarrow A^{4} \rightarrow I \rightarrow 0 .
$$

Note that $a+e$ is a regular element in $I$. By (1.5), we have $I=t D$ where $D=\left(d_{1}, d_{2}, d_{3}, d_{4}\right), t$ regular and $D^{-1}=A$. It is easy to see $d_{1}+d_{3}$ is a regular element in $D$, hence, by (1.3), $D$ is overdense. As (1.4) indicated, if we show $D \subset M$ then $M$ will be finitely generated. To check this, let us first note that $e \in Q, g \in P$ and $a=t d_{1}, b=t d_{2}, e=t d_{3}, g=t d_{4}$. Clearly $t \notin P$ or $t \notin Q$, as otherwise $a=t d_{1} \in Q$ or $b=t d_{2} \in P$ yields a contradiction. Since $P$ is a prime ideal and $a=t d_{1} \in P$, hence $d_{1} \in P \subset M$. Similarly, $d_{4} \in P$ and $d_{2}, d_{3} \in Q \subset M$.

If $A$ is a ring of global dimension two, the weak dimension of $A$ (wd $A$, for short) could be 0,1 or 2 . An immediate consequence of the preceding is

Corollary 2.1. If $\operatorname{gldim} A=\mathrm{wd} A=2$ and $A$ is coherent, there is a finitely generated maximal ideal. 
Proof. The condition wd $A=2$ amounts to saying that for some prime ideal $P, A_{P}$ is not a valuation domain. Since $A_{P}$ is of global dimension two it must then be either noetherian or an umbrella ring [11]. In either case we must have $P$ maximal containing two noncomparable primes and (1.1) applies.

3. A characterization of noetherian domains of global dimension two. In this section, $A$ will be, unless otherwise specified, a commutative ring of global dimension two.

A domain $A$ is called a Krull ring if $A$ satisfies the following two conditions: (1) If $P$ is a prime ideal of height one in $A$ then $A_{P}$ is a noetherian valuation ring. (2) An arbitrary principal ideal $a A$ of $A$ is the intersection of a finite number of primary ideals of height one.

Proposition 3.1. Let $A$ be a local ring of global dimension two, then $A$ is a Krull ring if and only if $A$ is noetherian.

Proof. First note $A$ is integrally closed [11]. It is well known that a noetherian integrally closed domain is a Krull ring [9]. Conversely, according to [11], it suffices to show that $A$ is neither a valuation domain nor an umbrella ring. Since a Krull ring with Krull dimension one is noetherian [9], assume that the Krull dimension of $A$ is at least two. Let $P$ be a prime ideal of $A$ which is not maximal; pick $x \notin P, x$ not a unit, hence $x A \not \subset P$. If $A$ is a valuation ring, then $P \subset x A=Q_{1} \cap \cdots \cap Q_{n} \subset$ $P_{1} \cap \cdots \cap P_{n}$ where $Q_{i}$ is $P_{i}$-primary and height of $P_{i}$ is one for all $i$. Hence $P \subset x A \subset P_{i}$ for some $i$, thus $x A=P$ which kills the fact that $A$ is a valuation ring. If $A$ is an umbrella ring, pick $P$ as a countably generated prime ideal (if no such $P$ then $A$ is noetherian), then $P \subset x A$ for some prime element $x \notin P[11]$, and the argument above contradicts $A$ is an umbrella ring.

THEOREM 3.2. Let A be a domain of global dimension two. If $A$ is a Krull ring then $A$ is noetherian.

Proof. We prove that every prime ideal $M$ is finitely generated. Notice that $A_{M}$ is a Krull ring [9] and, by (3.1), $A_{M}$ is a noetherian if $M$ is an ideal of height two, and by (1.1) $M$ is finitely generated.

Let $P$ be a prime ideal of height one; then $A_{P}$ is a discrete valuation ring with a maximal ideal generated by $a \in P$. Consider the primary decomposition of $a A$ :

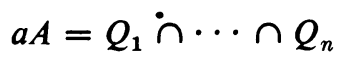

where $Q_{1}$ is the primary component corresponding to $P$. Pick $b \in Q_{2} \cap \cdots$ $\cap Q_{n}-P$; it then follows by the choices of $a$ and $b$ that $P=(a): b$, which is finitely generated since $A$ is coherent.

To end this section, consider the example of [8]. 
Let $F=K\left(a_{i}, b_{i}, i=1,2, \cdots\right)$ be an infinite pure transcendental extension with generators $a_{i}, b_{i}$ over the prime field of characteristic 2 . Let $F[[x, y]]=$ $R$ be the formal power series ring in indeterminates $x, y$ over $F$. Let $g$ be the automorphism of $R$ defined by

$$
\begin{gathered}
g(x)=x, \quad g(y)=y, \\
g\left(a_{i}\right)=a_{i}+y\left(a_{i+1} x+b_{i+1} y\right)^{-j} \text { and } g\left(b_{i}\right)=b_{i}+x\left(a_{i+1} x+b_{i+1} y\right) .
\end{gathered}
$$

Note that $g^{2}=1$. Let $A$ be the subring of $R$ of elements left fixed by $g$. By $[9$, p. 55], $A$ is a Krull ring. Since $A$ is not noetherian [8], by (3.2), $A$ is not a ring of global dimension two.

4. Finitely generated primes. Let $P$ be a finitely generated prime ideal of $A$. We begin with some observations on $A / P$. Since $\operatorname{pd}_{A} A / P \leqq 2, P$ is either projective or $\operatorname{pd}_{A} P=1$.

If $\operatorname{pd}_{A} P=0$ and the annihilator $I$ of $P$ is different from zero, then $P$ must be a minimal prime (otherwise $I$ would be contained in each minimal prime and would be a nilideal, which is impossible since $A$ is reduced). In this case, since $P$ is a pure ideal, $A / P$ is a ring of global dimension at most two and thus coherent. Note that in this case $P$ is generated by one idempotent.

Let us then assume that $P$ is a faithful ideal and consider two cases depending on whether the projective dimension of $P$ is 0 or 1 .

We begin by remarking that if $A$ is a ring of global dimension two (not necessarily coherent) and $I$ is a finitely generated faithful ideal then $A / I$ is coherent. Indeed, let $J$ be a finitely generated ideal above $I ; J$ being faithful it is of finite presentation. The sequence

$$
0 \rightarrow I \rightarrow J \rightarrow J / I \rightarrow 0
$$

then says (by Schanuel's lemma) that $J / I$ has finite presentation as an $A$-module. Thus there is an exact sequence

$$
A^{m} \rightarrow A^{n} \rightarrow J / I \rightarrow 0
$$

which by tensorization with $A / I$ gives the desired conclusion.

Case I. $\operatorname{pd}_{A} P=0$. We show that $A / P$ is noetherian.

We recall the definition of the finitistic projective dimension of a ring $B$ (FPD $B$, for short), is simply obtained by considering in the definition of the global dimension only those modules with finite projective dimension.

Proposition 4.1. Let $B$ be a coherent domain with FPD $B \leqq 1$. Then $B$ is noetherian. 
Proof. If the finitistic projective dimension of a coherent ring $B$ is zero, then $B$ is artinian [3] and thus $B$ is noetherian. If FPD $B=1$, let $Q$ be a nontrivial prime ideal of $B$ and pick $0 \neq a \in Q$. The ring $B / a B$ has, by the change of ring theorem of [7], FPD $B / a B=0$. As $B / a B$ is also coherent, it follows from the above that $B / a B$ is noetherian and hence $Q$ is finitely generated.

The ring $A / P$ has, by the change of ring theorem of [4], [5], finitistic dimension at most one and hence, by (4.1), $A / P$ is noetherian.

Case II. $\operatorname{pd}_{A} P=1$. Since $P$ has a finite presentation, $\operatorname{pd}_{A} P=\mathrm{pd}_{A_{Q}} P_{Q}$ for some maximal ideal $Q$. But in $A_{Q}$, by [11], the only finitely generated prime ideal with projective dimension one is the maximal ideal; thus $P=Q$.

5. Estimating the number of generators. We would like to estimate the number of generators of a finitely generated prime ideal $P$ of $A$ in this section.

If $P$ is a minimal prime, we have already seen that it is generated by one idempotent.

Let us again assume $P$ faithful and consider two cases.

Case I. $P$ is projective. $P \otimes A / P=P / P^{2}$ is $A / P$-projective of rank one; as $A / P$ is a domain, $P / P^{2}$ can be viewed as an ideal of $A / P$. Since $A / P$ is a one dimensional domain, $P / P^{2}$, by [13], is generated by two elements and $P$ is generated by three elements [13].

Case II. $P$ is a maximal ideal. We show that $P$ is generated by three elements if $A$ is coherent of global dimension two.

We may assume that $P_{P}$ is not principal; then $P_{P}$ is generated by two elements [11], say $a, b$. Notice that we may pick $a$ to be a regular element since the generator $e$ of the annihilator of $a$ is also in $P$ and we could equally consider $a+e$ instead of $a$. Let $I=(a, b) \subset P$; Then $I=Q J$ with $Q$ projective and $J$ overdense [12]. Thus FPD $A / J=0$ and $A / J$ is artinian; $J$ is then contained in only finitely many maximal ideals, say $P, P_{1}, \cdots, P_{n}$. Since $Q \not \subset P$ (check the localization $A_{P}$ ), we have $Q P_{1} \cdots P_{n}+P=A$, i.e. an equation $x+c=1$ where $x \in Q P_{1} \cdots P_{n}$ and $c \in P$. We claim $(a, b, c)=$ $P$. We prove the claim by localization:

Let $M$ be a maximal ideal. If $(a, b) \notin M$, we are done. If $Q \subset M, c \notin M$ and again we get that $(a, b, c)$ blows up at $M$. Finally, if $J \subset M, M$ must be one of the ideals $P, P_{1}, \cdots, P_{n}$. If $M=P$ the choices of $a, b$ take over, while in the other cases $c$ lies outside $M$. This completes the proof.

It is likely that the coherence of $A$ is redundant for the validity of Case II.

\section{REFERENCES}

1. M. Auslander, On the dimension of modules and algebras. III. Global dimension, Nagoya Math. J. 9 (1955), 67-77. MR 17, 579. 
2. N. Bourbaki, Eléments de mathématique, Fasc. XXVII. Algèbre commutative. Chap. 1: Modules plats. Chap. 2: Localisation, Actualités Sci. Indust., no. 1290, Hermann, Paris, 1961. MR 36 \#146.

3. S. U. Chase, Direct product of modules, Trans. Amer. Math. Soc. 97 (1960), 457-473. MR 22 \#11017.

4. J. M. Cohen, A note on homological dimension, J. Algebra 11 (1969), 483-487.

5. C. U. Jensen, Some remarks on a change of rings theorem, Math. Z. 106 (1968), 395-401.

6. I. Kaplansky, Commutative rings, Allyn and Bacon, Boston, Mass., 1970. MR 40 \#7234.

7. ___ Fields and rings, Univ. of Chicago Press, Chicago, Ill., 1969. MR 42 \#4345.

8. K. R. Nagarajan, Groups acting on noetherian rings. Nieuw Arch. Wisk. (3) 16 (1968), 25-29. MR 37 \#5202.

9. P. Samuel, Lectures on unique factorization domains, Tata Institute of Fundamental Research Lectures on Math., no. 30, Tata Institute of Fundamental Research, Bombay, 1964. MR 35 \#5428.

10. W. V. Vasconcelos, On finitely generated flat modules, Trans. Amer. Math. Soc. 138 (1969), 505-512. MR 39 \#199.

11. - The local rings of global dimension two, Proc. Amer. Math. Soc. 35 (1972), 381-386.

12. — Rings of global dimension two, Proc. Comm. Algebra Conf., Lecture Notes in Math., vol. 311, Springer-Verlag, New York, and Berlin, 1972.

13. —, Finiteness in projective ideals, J. Algebra (to appear).

14. - Coherence of one polynomial ring, Rutgers University (preprint).

Department of Mathematics, Rutgers University, New Brunswick, New JERSEY 08903 